\title{
The Control of Mimicry by Eye Contact Is Mediated by Medial Prefrontal Cortex
}

\author{
Yin Wang, Richard Ramsey, and Antonia F. de C. Hamilton \\ School of Psychology, the University of Nottingham, Nottingham NG7 2RD, United Kingdom
}

\begin{abstract}
Spontaneous mimicry of other people's actions serves an important social function, enhancing affiliation and social interaction. This mimicry can be subtly modulated by different social contexts. We recently found behavioral evidence that direct eye gaze rapidly and specifically enhances mimicry of intransitive hand movements (Wang et al., 2011). Based on past findings linking medial prefrontal cortex (mPFC) to both eye contact and the control of mimicry, we hypothesized that $\mathrm{mPFC}$ might be the neural origin of this behavioral effect. The present study aimed to test this hypothesis. During functional magnetic resonance imaging (fMRI) scanning, 20 human participants performed a simple mimicry or no-mimicry task, as previously described (Wang et al., 2011), with direct gaze present on half of the trials. As predicted, fMRI results showed that performing the task activated mirror systems, while direct gaze and inhibition of the natural tendency to mimic both engaged mPFC. Critically, we found an interaction between mimicry and eye contact in mPFC, superior temporal sulcus (STS) and inferior frontal gyrus. We then used dynamic causal modeling to contrast 12 possible models of information processing in this network. Results supported a model in which eye contact controls mimicry by modulating the connection strength from mPFC to STS. This suggests that mPFC is the originator of the gaze-mimicry interaction and that it modulates sensory input to the mirror system. Thus, our results demonstrate how different components of the social brain work together to on-line control mimicry according to the social context.
\end{abstract}

\section{Introduction}

Human behavior depends critically on social contexts, a sensitivity based on a series of rapid and automatic processes such as gaze perception, emotion detection, and action mimicry (Adolphs, 2009). However, little is known about the interplay between these processes and how they interact in the brain. We recently found behavioral evidence that action mimicry can be directly modulated by eye contact: direct gaze rapidly and specifically enhances the mimicry of hand movements compared with averted gaze (Wang et al., 2011). The aim of this study is to use functional magnetic resonance imaging ( $\mathrm{fMRI}$ ) to investigate the neural mechanism of this effect.

Mimicry refers to the unconscious tendency to copy the postures, gestures, and mannerisms of others (Chartrand and van Baaren, 2009). It has been suggested that this spontaneous mimicry acts as a "social glue," and increases affiliation and liking between interaction partners (Chartrand and van Baaren, 2009). As a form of imitation, mimicry has been strongly associated with the mirror neuron system (MNS) (Iacoboni, 2009). This brain network spans inferior frontal gyrus (IFG) and inferior parietal

Received Feb. 16, 2011; revised June 23, 2011; accepted June 28, 2011.

Author contributions: Y.W. and A.F.C.H. designed research; Y.W. performed research; Y.W., R.R., and A.F.C.H. analyzed data; Y.W., R.R., and A.F.C.H. wrote the paper.

Y.W. is supported by Overseas Research Students Award and University of Nottingham Scholarship. This work was also supported by Economic and Social Research Council Grant RES-061-25-0138 (to A.H.). We thank Chris Frith for helpful comments on the manuscript.

Correspondence should be addressed to Yin Wang, School of Psychology, the University of Nottingham, University Park, Nottingham NG7 2RD, UK. E-mail: mirrorneuronwang@gmail.com.

DOI:10.1523/JNEUROSCI.0845-11.2011

Copyright $\odot 2011$ the authors $\quad 0270-6474 / 11 / 3112001-10 \$ 15.00 / 0$ lobule (IPL), and is engaged in a variety of imitation and imitation learning tasks (Iacoboni et al., 1999; Buccino et al., 2004; Rizzolatti and Craighero, 2004; Iacoboni, 2009). It is claimed that this network implements a "direct-mapping mechanism" that matches an observed action to a motor representation of that action (Rizzolatti and Craighero, 2004; Brass and Heyes, 2005).

However, this direct mapping of observed actions to performed actions is not impervious to other processes, but can be flexibly modulated by higher-level cognitive processes such as intention attribution and social relevance observation (Kilner et al., 2006; Liepelt et al., 2008; Liepelt and Brass, 2010). Similarly, studies of mimicry in social contexts also emphasize the importance of controlling when and who to mimic. Attractiveness, friendship, and social status can enhance mimicry while social stigma, negative mood, and outgroup membership can inhibit mimicry (Chartrand and van Baaren, 2009).

One candidate brain system for the control of mimicry by social contexts is medial prefrontal cortex (mPFC). mPFC is considered as a core region for social cognition (Amodio and Frith, 2006). It is engaged when detecting and evaluating direct gaze (Kampe et al., 2003; Kuzmanovic et al., 2009). Brass et al. (2001, 2005, 2009) suggest that mPFC is engaged when participants must inhibit their natural tendency to mimic, and patient studies suggest damage to prefrontal cortex can lead to overimitation (Luria, 1980). This evidence makes mPFC a strong candidate for controlling the interaction of gaze and mimicry. Alternatively, the control of mimicry by gaze might be linked to superior temporal sulcus (STS), a key center for processing of eye gaze (Senju and Johnson, 2009) and a sensory input site to the MNS (Rizzolatti and Craighero, 2004). In the present study, we used our 
established paradigm (Wang et al., 2011) to identify brain systems involved in the control of mimicry by eye contact, using both a factorial fMRI event-related design and dynamic causal modeling (DCM).

\section{Materials and Methods}

Participants. Twenty paid participants (5 males, 15 females; mean \pm SD age, $23 \pm 4.8$ years) were recruited for the present study. All participants were right handed, with normal or corrected-tonormal vision and no history of brain damage. They gave their informed consent to complete the experiment, in accord with the local ethics board.

Stimuli and experimental design. In each trial, participants watched a video clip where an actress performed an eye/head movement and a hand movement (Fig. 1). At the onset of the video clip, the actress was facing away from the camera, with her eyes closed and her left hand still in front of her face. Then she opened her eyes and naturally moved her head either toward the camera, which resulted in a direct gaze or a gaze toward her left/right side, which resulted in an averted gaze; her hand remained entirely still. Subsequently, the actress performed a hand movement. She either opened her hand or closed her hand (stimulus trials), or remained hand static (catch trials). All stimuli were identical to Wang et al. (2011) and were presented with Cogent toolbox running under Matlab 6.5, permitting synchronization with the scanner and accurate timing of stimuli presentation.

We used a stimulus-response compatibility paradigm as before (Heyes et al., 2005; Press et al., 2008; Wang et al., 2011). For each block, participants were required to make the same prespecified response in every trial. They had to always open or close their right hand as quickly as possible after the actress' hand in video clips began to move. On some trials, the actress' hand opened, and on others it closed. Therefore, within a block, the hand movement in the movie was either the same as the prespecified response (congruent trials: e.g., open stimulus and open response) or the opposite of the prespecified response (incongruent trials: e.g., close stimulus and open response). Past studies have found a clear congruency effect (CE) in this paradigm and took CE as a reliable measure of mimicry, with faster responses on congruent trials, which were facilitated by mimicry of observed congruent action, and slower responses on incongruent trials, where participants must inhibit the natural tendency to mimic the actress' hand action (Heyes et al., 2005; Press et al., 2008; Heyes, 2011). Our previous study demonstrated that this CE can be enhanced by eye contact where direct gaze significantly reduces reaction times on congruent trials (Wang et al., 2011). Compliance with the hand movement task was monitored from the scanner control room. For technical reasons, it was not possible to measure precise reaction times during fMRI, but as the behavioral result has now been replicated four times in different participant groups (Wang et al., 2011: experiment 1 and experiment 2, and two pilot studies), we are confident that it is robust.

To prevent anticipatory responding, there was a variable delay (200 or $800 \mathrm{~ms}$ ) between the end of the actress' head movement and the start of her hand movement in the video, and in addition $\sim 20 \%$ of trials in a block were catch trials (Wang et al., 2011). In catch trials, the hand observed in the video remained static. Participants were instructed to refrain from moving their right hand, but were asked to detect a small white box that appeared on the top of the still hand and press a response button with their left hand when the white box was detected. Thus, participants mimicked the actress' hand movements only in stimulus trials (congruent and incongruent), but not in catch trials. Participants were trained on all tasks (congruent, incongruent, and catch) for $\sim 5 \mathrm{~min}$ before the fMRI measurement.

We adopted a $2 \times 3$ factorial event-related design in which the factors were "Gaze Direction" (two levels: direct or averted gaze) and "Action
Congruency" (three levels: congruent or incongruent, or catch trials) (Fig. 1). Each trial was defined by these two factors as direct gaze with congruent action (Direct-Cong), direct gaze with incongruent action (Direct-Incong), direct gaze with catch action (Direct-Catch), averted gaze with congruent action (Averted-Cong), averted gaze with incongruent action (Averted-Incong), and averted gaze with catch action (AvertedCatch). There were six blocks; three blocks required a hand-close response, and three blocks required a hand-open response. Block order alternated and was randomized across participants. Each block presented in a pseudorandom order 32 stimulus trials (16 congruent and 16 incongruent) and 9 catch trials. The first trial in each block was always a catch trial and was excluded from further analysis.

fMRI data acquisition. Subjects lay supine on the scanner bed, with their right hand uprightly positioned on the abdomen and their left hand positioned over the fMRI button box. A participant's right hand was carefully stabilized, and form-fitting cushions were used to prevent arm, hand, and head motion. To attenuate scanner noise, participants were provided with earplugs.

Imaging was performed using a 3T Philips Achieva scanner, equipped with an eight channel phased-array head coil. Thirty-eight axial slices (field of view: $240 \times 240 \mathrm{~mm}^{2}$; matrix: $80 \times 80$; thickness: $3 \mathrm{~mm}$ ) parallel to the bicommissural line (anterior commissure-posterior commissure) were acquired using a T2*-weighted gradient EPI sequence (TR: $2500 \mathrm{~ms}$; TE: $40 \mathrm{~ms}$; flip angle: $80^{\circ}$ ). Before the functional runs, structure images were also required for each participant using a high-resolution, T1weighted MPRAGE sequence.

Conventional general linear model analysis. To remove sources of noise and artifact, functional data were realigned, unwarped, corrected for slice timing, normalized to the MNI template with a resolution of $3 \times$ $3 \times 3 \mathrm{~mm}$, and spatially smoothed $(8 \mathrm{~mm})$ using SPM8 software. A design matrix was fitted for each subject with one regressor for each movie type (Direct-Cong, Direct-Incong, Direct-Catch, Averted-Cong, Averted-Incong, and Averted-Catch) and combined across the six blocks. Each movie was modeled as a boxcar with the duration of that movie convolved with the standard hemodynamic response function. To reduce the influence of reaction time variability between conditions on the general linear model (GLM) analysis (Grinband et al., 2008), we added an extra column in the design matrix, modeling our previous average reaction time scores (Wang et al., 2011, study 1) in each condition as a parametric regressor.

To localize brain regions engaged in the hand movement task, two contrasts (Congruent trials $>$ Catch trials and Incongruent trials $>$ Catch trials) were calculated across all movies. To localize brain regions for inhibition of mimicry, a contrast for the main effect of mimicry inhibition (Incongruent trials $>$ Congruent trials) was calculated. Two contrasts for the simple effect of the inhibition of mimicry were also calculated 
Table 1. Cortical activation for the hand movement task, inhibition of mimicry, eye contact effect, and the interaction between mimicry and eye contact

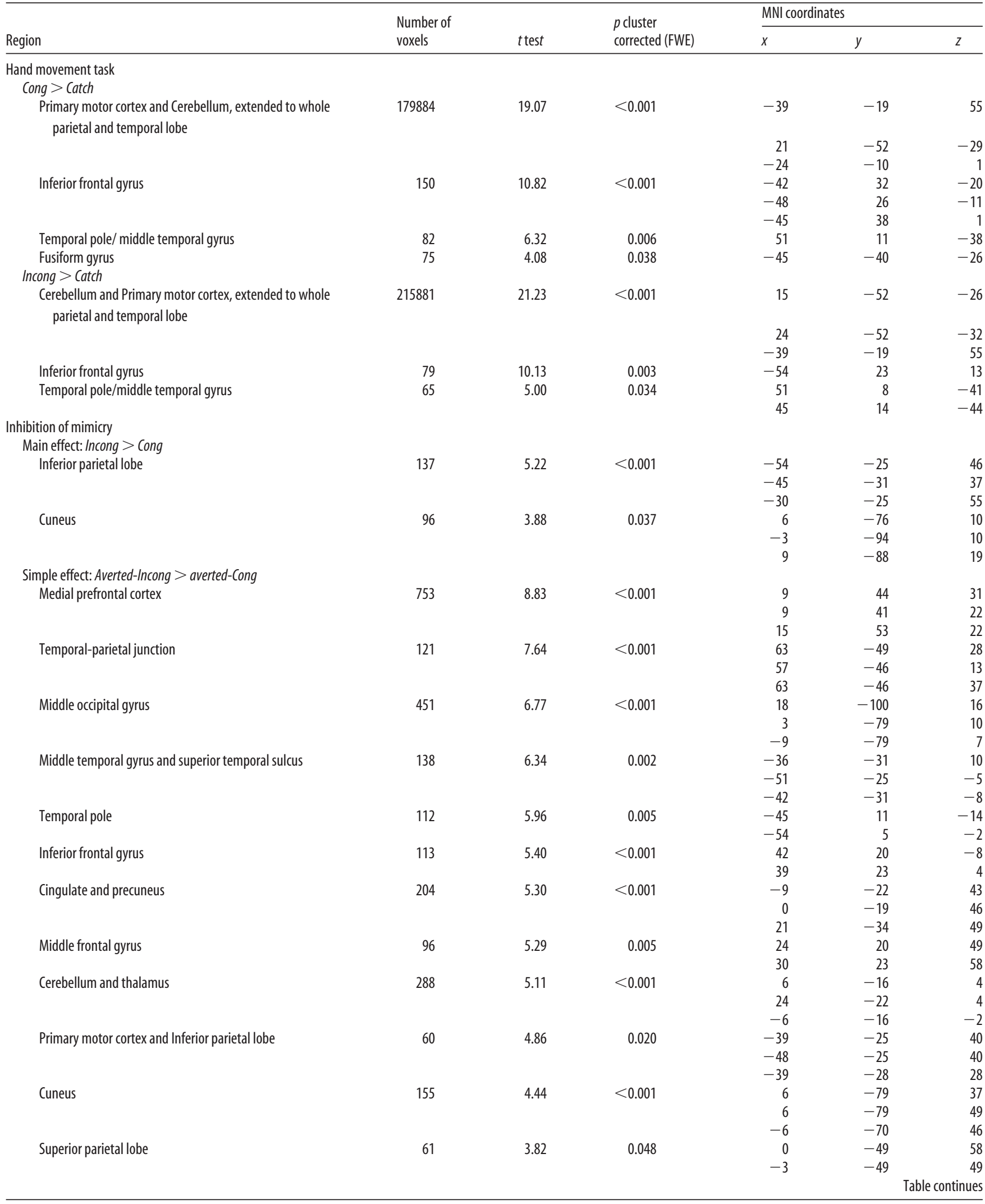


Table 1. Continued

\begin{tabular}{|c|c|c|c|c|c|c|}
\hline \multirow[b]{2}{*}{ Region } & \multirow{2}{*}{$\begin{array}{l}\text { Number of } \\
\text { voxels }\end{array}$} & \multirow[b]{2}{*}{$t$ test } & \multirow{2}{*}{$\begin{array}{l}p \text { cluster } \\
\text { corrected (FWE) }\end{array}$} & \multicolumn{3}{|c|}{ MNI coordinates } \\
\hline & & & & $x$ & $y$ & $z$ \\
\hline \multicolumn{7}{|l|}{ Eye contact effect } \\
\hline \multirow[t]{2}{*}{ Medial prefrontal cortex } & 45 & 7.37 & $<0.001$ & 9 & -10 & 1 \\
\hline & 47 & 5.64 & $<0.001$ & 15 & 44 & 13 \\
\hline \multirow[t]{2}{*}{ Superior temporal sulcus/ Middle temporal gyrus } & 77 & 7.19 & $<0.001$ & 48 & -46 & 1 \\
\hline & & & & 48 & -40 & -5 \\
\hline \multirow{2}{*}{ Inferior parietal lobe } & & & & 54 & -49 & 49 \\
\hline & & & & 45 & -70 & 40 \\
\hline \multicolumn{7}{|l|}{ Interaction } \\
\hline \multicolumn{7}{|c|}{ (Direct-Cong $>$ Direct-Incong) $>$ (Averted-Cong $>$ Averted-Incong) } \\
\hline \multirow[t]{2}{*}{ Superior temporal sulcus } & 53 & 7.12 & $<0.001$ & -48 & -19 & -2 \\
\hline & & & & -51 & -28 & -2 \\
\hline \multirow{2}{*}{ Inferior frontal gyrus } & & & & 39 & 26 & -23 \\
\hline & & & & 24 & 14 & -26 \\
\hline
\end{tabular}

Only regions surviving a whole-brain voxel-level threshold of $p<0.001$ and 30 voxels and a FWE cluster-corrected level threshold of $p<0.05$ are reported. Subpeaks $>8 \mathrm{~mm}$ from the main peak in each cluster are listed.

(Direct-Incong $>$ Direct-Cong and Averted-Incong $>$ Averted-Cong). To identify brain regions that code the eye contact effect, a contrast for the main effect of eye contact (Direct $>$ Averted) was performed across all movies. Also, two contrasts for the simple effect of eye contact were calculated across all movies of stimulus trials (Direct-Cong $>$ AvertedCong and Direct-Incong $>$ Averted-Incong). Finally, we calculated the interaction between mimicry and eye contact, both as (Direct-Cong $>$ Direct-Incong) $>$ (Averted-Cong $>$ Averted-Incong) and as the inverse contrast (Direct-Incong $>$ Direct-Cong) $>$ (Averted-Incong $>$ AvertedCong). Contrast images for all participants were then taken to the second level for a random-effects (RFX) analysis in SPM8. Brain regions were initially thresholded at a voxel-level threshold of $p<0.001$ and 30 voxels. Only regions that survive a cluster-level familywise error (FWE) correction of $p<0.05$ over the whole brain are discussed and reported in Table 1 and Figure 2.

$D C M$. To explore information processing between brain areas under different experimental manipulations, we performed an effective connectivity analysis using DCM10 (Friston et al., 2003). DCM treats the brain as a dynamic input-state-output system. The inputs correspond to experimental manipulations. The state variables are neuronal activities (firing rates), and the outputs are the regional hemodynamic responses measured with fMRI. The idea is to model changes in the hidden states and effective connectivity, which cannot be observed by fMRI directly, using the known inputs and outputs. The following three kinds of coupling parameters are estimated in DCM: (1) direct, extrinsic inputs to the system (i.e., the direct effect of eye contact on mPFC); (2) "intrinsic" or "fixed" connections that couple neuronal states between regions (i.e., the connectivity strength from mPFC to STS); and (3) modulatory parameters that model the changes in fixed connectivity induced by the experimental manipulations (i.e., the additive change a certain manipulation, like direct gaze, has on the strength of a connection).

For each participant, models were constructed to define the connections between and the inputs to three regions identified in the mimicrygaze interaction contrast (Fig. $2 d$ ). These were as follows: (1) the left STS as the "sensory input" of MNS $(x=-48, y=-19, z=-2$; coordinates are from Table 1); (2) the right IFG as the "motor output" of MNS ( $x=$ $45, y=26, z=-17)$; and (3) the right $\mathrm{mPFC}$ as a potential control region $(x=6, y=44, z=34)$. Input data to the models were extracted in a participant-specific manner from each of these three regions (Fig. 3a). In detail, the region-specific time series (concatenated over the six blocks) comprised the first eigenvariate of all voxels within a 5-mm-radius sphere centered on the subject-specific peak in the interaction contrast. The subject-specific peak was constrained within a 15 -mm-radius sphere centered on the peak coordinates from the group random-effect analysis (Fig. $3 a$ ). Of the 20 participants, we could not identify an individual peak in the STS in one participant, in the IFG in one participant, and in the $\mathrm{mPFC}$ in one participant. Thus, the data from these three participants were excluded from the DCM analysis, leaving 17 participants in this analysis.

The three regions in each model were set to be bidirectionally connected, according to anatomical evidence from human and monkey (Fig. $3 b$ ). For simplicity (Stephan et al., 2010), a new design matrix was created for DCM analysis that modeled the following two critical factors: (1) the hand movement task (stimulus trials vs catch trials); and (2) the interaction of gaze and mimicry (Direct-Cong $>$ Direct-Incong vs AvertedCong $>$ Averted-Incong) as parametric factors on the individual trials. This allows us to consider only these two factors as extrinsic inputs in the current study and substantially simplifies our original $2 \times 3$ factorial design. Holding the number of parameters constant for the intrinsic connectivity structure, extrinsic inputs, and modulatory effects, we compared $12=2 \times 3 \times 2$ models to address the following three central questions (Fig. $3 d$ ). (1) Is the eye contact effect on mimicry due to topdown modulation from mPFC (model 1-6) or bottom-up gating from STS (models 7-12)? (2) How does the interaction of gaze and mimicry modulate processing within the network? Is it by modulating the connection strength between mPFC and STS (models $1,4,7$, and 10), by modulating the connection strength from mPFC to IFG (models 2, 5, 8, and 11 ), or by modulating the connection strength from STS to IFG (models $3,6,9$, and 12)? (3) Does the hand movement task affect the connection strength from STS to IFG (models 4, 5, 6, 10, 11, and 12) or not (models $1,2,3,7,8$, and 9$)$ ?

Bayesian models selection. To determine the most likely of the 12 models given the observed data from all subjects, we implemented a fixed-effects (FFX) and an RFX group analysis (Stephan et al., 2009). In the FFX case, one assumes that the optimal model is identical across the population. It uses group log evidence to quantify the relative goodness of models, which is the exponentiated sum of the log model evidence of each subject-specific model (Penny et al., 2004). As the log evidence of each subject-specific model estimated by the FFX group analysis depends not only on model fit but also on model complexity, we limited ourselves to the 12 models that were equated for the number of parameters. Usually, a difference in group log evidence of three is taken as statistically strong evidence (Kass and Raftery, 1995). Thus, if the group log evidence of one model is bigger than the other models' by three or 


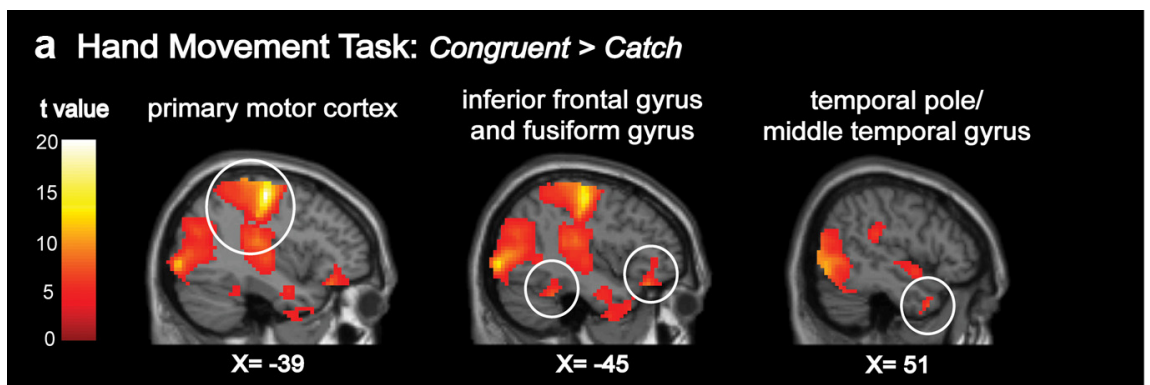

b Inhibition of Mimicry: Averted-Incong > Averted-Cong
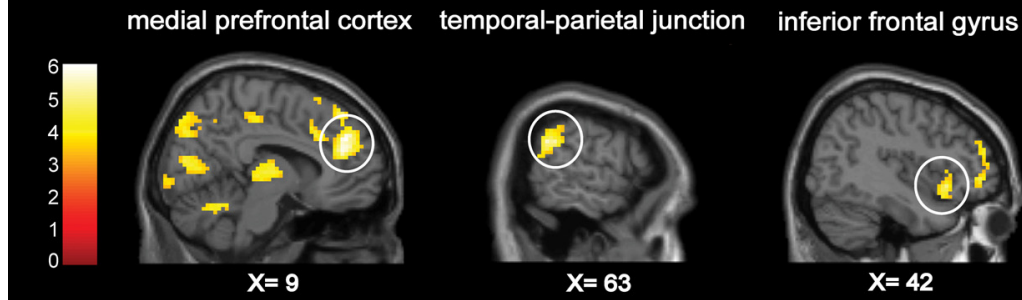

C Eye Contact Effect: Direct-Cong > Averted-Cong

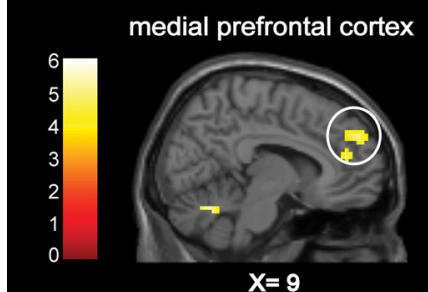

inferior parietal lobe
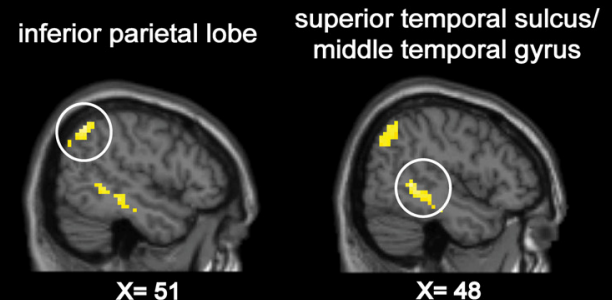

d Interaction: (Direct-Cong > Direct-Incong) > (Averted-Cong > Averted-Incong)
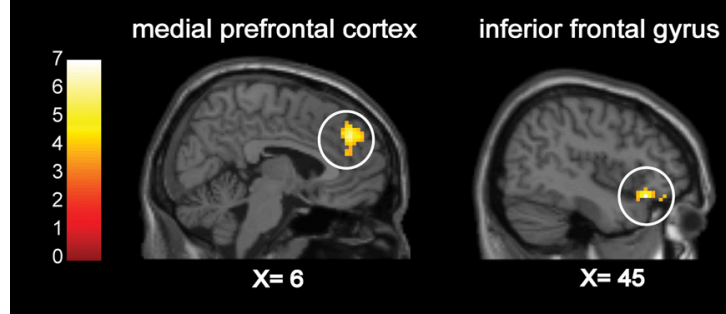

superior temporal gyrus
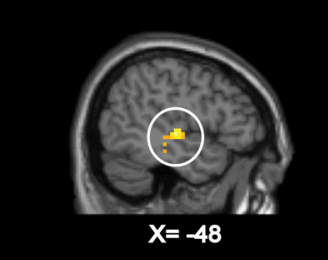

Figure 2. Brain regions showing increased activations for the hand movement task $(\boldsymbol{a})$, the simple effect of inhibition of mimicry $(\boldsymbol{b})$, the simple effect of eye contact $(\boldsymbol{c})$, and the interaction of gaze and mimicry $(\boldsymbol{d})$. Coordinates are listed in Table 1.

\section{Results}

Neural correlates of the hand movement task

The two contrasts Congruent trials $>$ Catch trials, Incongruent trials $>$ Catch trials both yielded strong activations in primary motor cortex, cerebellum, IFG, and temporal pole/middle temporal gyrus (MTG). Activations also extended to large areas in parietal and temporal lobe (Table 1, Fig. 2a).

\section{Neural correlates of inhibition of mimicry}

We examined brain regions engaged when participants must inhibit their natural tendency to mimic the observed hand action (Incongruent $>$ Congruent). Regardless of gaze direction, this main effect revealed greater activation in two regions: IPL and cuneus. We further examined the simple effect of the inhibition of mimicry in either direct or averted gaze conditions. Specifically, when mimicry was preceded by an averted gaze, brain areas that showed greater response to "incongruent" trials than "congruent" trials (the contrast Averted-Incong > Averted-Cong) included $\mathrm{mPFC}$, temporal-parietal junction (TPJ), middle occipital gyrus, MTG/STS, IFG, middle frontal gyrus (MFG), IPL, cingulated, and precuneus (Table 1 and Fig. $2 b$ ). This result replicates the findings from Brass et al. $(2001,2005,2009)$ that mPFC and TPJ have a role in inhibition of mimicry. However, no region was found to show greater activation to incongruent trials than congruent trials in direct gaze conditions (the contrast Direct-Incong $>$ Direct-Cong).

Neural correlates of the eye contact effect We examined the brain regions responsive to direct gaze compared with averted gaze (main effect, Direct $>$ Averted). No regions survived our thresholds in this contrast. We then separately examined more, that model would be considered by FFX analysis to be the optimal model (Stephan et al., 2010).

Because the FFX analysis is vulnerable to outlier subjects, we also implemented an RFX analysis, which accounts for the heterogeneity of the model structure across subjects (Stephan et al., 2009). It uses hierarchical Bayesian modeling that estimates the parameters of a Dirichlet distribution over the probabilities of all models considered. These probabilities define a multinomial distribution over model space, enabling the computation of the posterior probability of each model given the data of all subjects and the models considered. The results of RFX analysis are reported in terms of the exceedance probability that one model is more likely than any other model. The optimal model in RFX analysis would be considered to be the one with the largest exceedance probability as well as the one that is above chance level.

For the optimal models selected by FFX and RFX analysis, the model parameters (intrinsic connection strength, effects of extrinsic inputs, and modulatory effects of extrinsic inputs on connection strength) were entered into $t$ tests at the group level. This allowed us to summarize the consistent findings from the subject-specific models using classical statistics. the simple effect of eye contact in either congruent action conditions or incongruent conditions. The contrast Direct-Cong $>$ Averted-Cong revealed increased neural activity in MTG/STS, IPL, and mPFC (Table 1, Fig. 2c). This result replicates the findings from Kampe et al. (2003) that mPFC is engaged by eye Incong $>$ Averted-Incong.

\section{Neural correlates of interaction between mimicry and eye contact}

Results from the interaction contrast (Direct-Cong $>$ Direct-Incong) $>$ (Averted-Cong $>$ Averted-Incong) revealed activity in the following three areas: the MPFC, IFG, and STS (Table 1, Fig. 2d). Illustrative plots of the parameter estimates in $\mathrm{mPFC}$ revealed strong engagement of this region in the Direct-Cong and AvertedIncong conditions (Fig. 4c). Further plots of the relationship between this interaction contrast and the simple effect of eye contact (congruent trials only) and the simple effect of the concontact. No brain region was found in the other contrast Direct- 


\section{a Individual ROI selection}
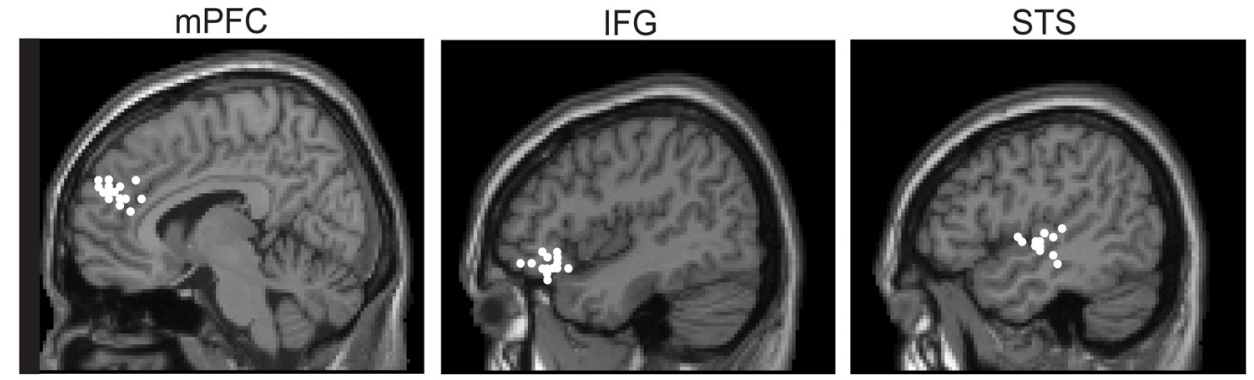

\section{b Anatonial Connectivity: summary of the available evidence on the basis of tracer studies in monkeys and DTI studies on human}

\begin{tabular}{|c|c|}
\hline Connection & References \\
\hline STS & Rilling et al 2008 . Gong et al 2009 \\
\hline $\mathrm{mPFC} \leftrightharpoons \mathrm{STS}$ & Leichnetz et al.,1976; Carmichael et al.,1995; Bachevalie \\
\hline $\mathrm{mPFC} \leftrightharpoons \mathrm{IFG}$ & Carmichael et al.,1995; Luppino et al.,2003; Gong et al., 2009 \\
\hline
\end{tabular}

\section{c DCM design matrix}

\section{d Model space}
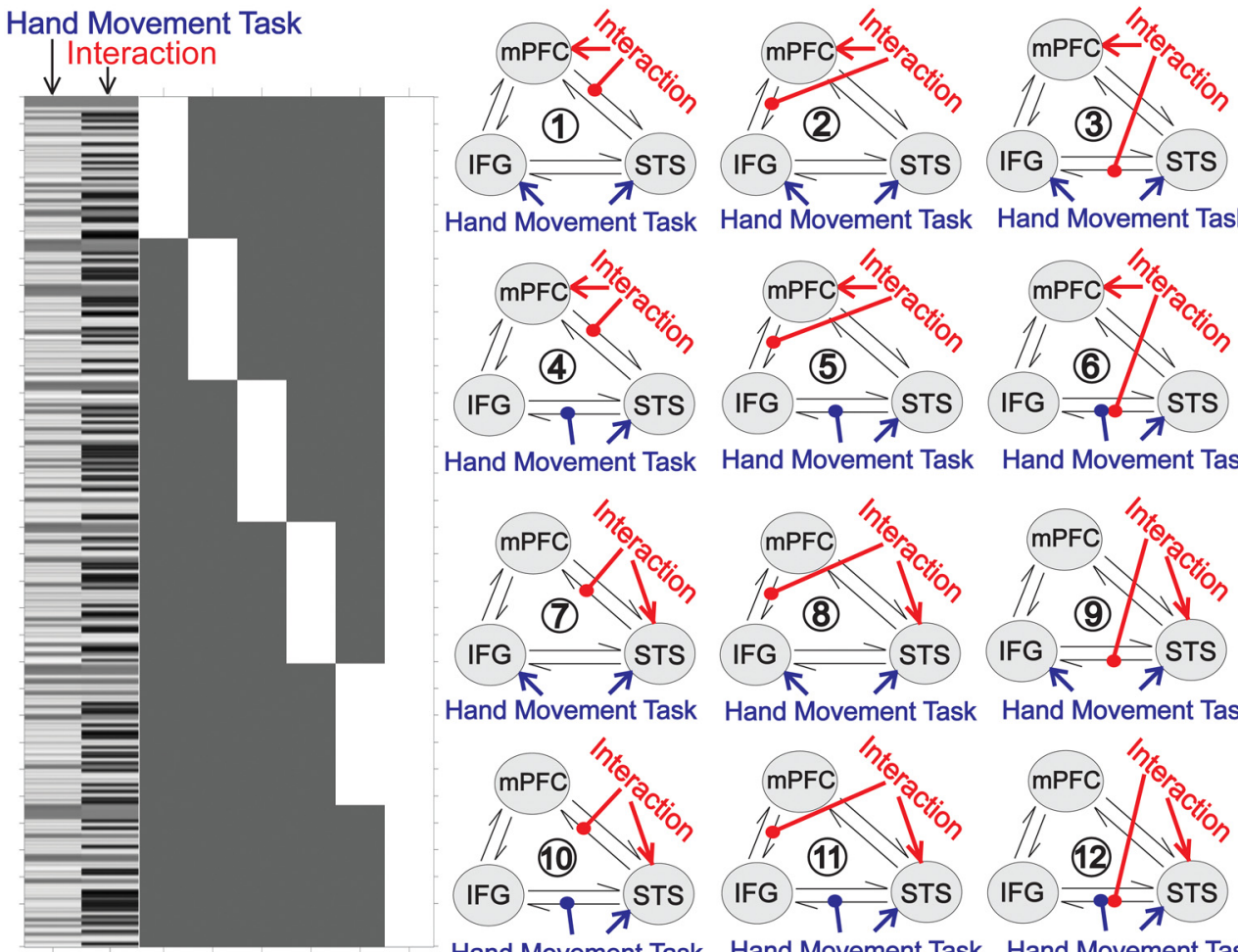

Hand Movement Task
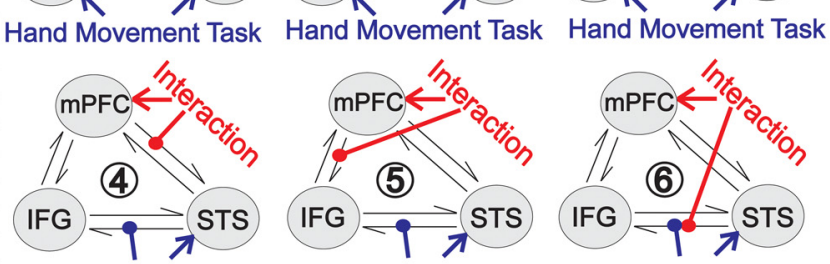

Hand Movement Task

Hand Movement Task

Hand Movement Task
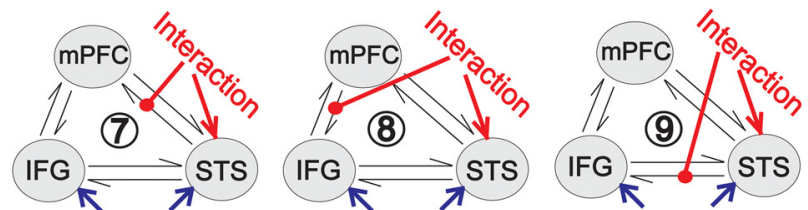

Hand Movement Task

Hand Movement Task

Hand Movement Task
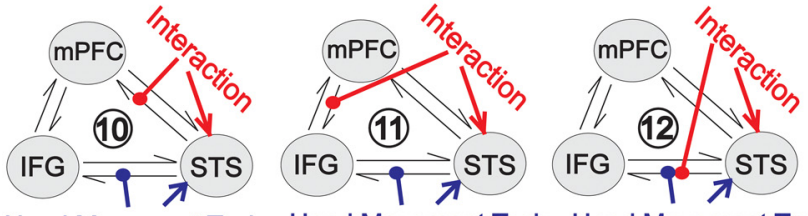

Hand Movement Task Hand Movement Task Hand Movement Task

Figure 3. DCM inputs. $\boldsymbol{a}$, Region selection and time series extraction from 17 subjects. Each white point indicates the center of the $5 \mathrm{~mm}$ radius sphere where data were extracted for one participant. $\boldsymbol{b}$, Anatomical criteria used to define the intrinsic connectivity structure. $\boldsymbol{c}$, The new matrix design for DCM. Only two critical factors were analyzed in DCM: hand movement task and interaction. $\boldsymbol{d}$, The model space of all 12 models was considered in the DCM analysis.

trol of mimicry (averted gaze only) revealed that all three contrasts overlapped in mPFC (Fig. 4a). The inverse contrast (DirectIncong $>$ Direct-Cong $)>($ Averted-Incong $>$ Averted-Cong $)$ did not yield any significant clusters.

DCM and Bayesian models selection results

Figure $5 a$, right, shows the relative group log evidence across the 12 models and the structure of the best model (model 4 ). The relative group log evidence refers to log evidence summed over 17 subjects for 12 models relative to the worst model. The FFX group analysis provided strong evidence for model 4 , as the difference of the relative group log evidence between model 4 and the second best model (model 10) was 3.52. The RFX analysis on the same set of models gave a compatible result, with model 4 providing the best description of the observed data. As shown in Figure $5 b$, right, model 4 was associated with the largest model exceedance probability of 0.195 , which is much more than the exceedance probability of $1 / 12=0.083$ when assuming a uniform distribution over models. As the exceedance probability of a particular model in the RFX analysis does not only depend on the data but 


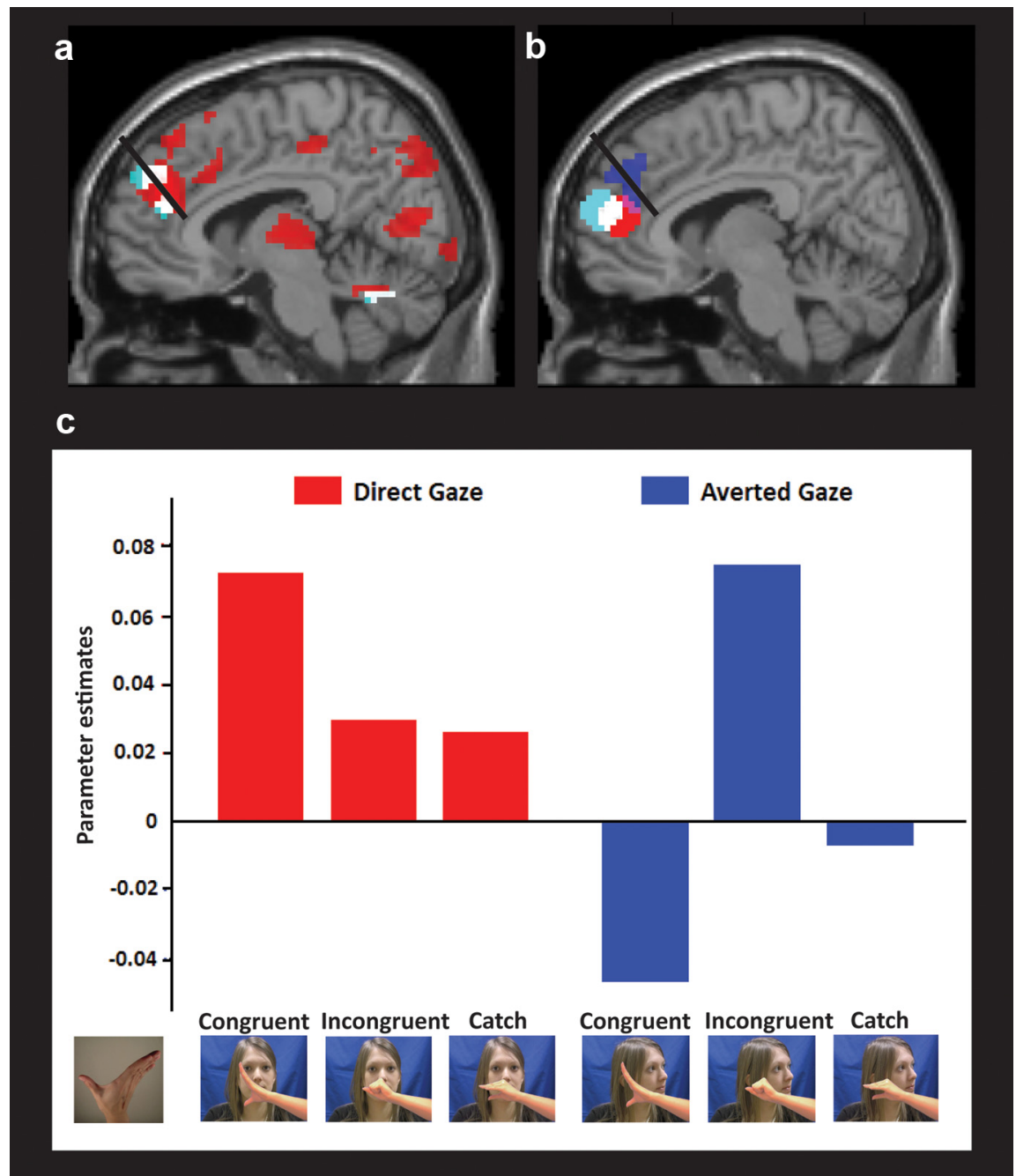

Figure 4. Activations in mPFC. $\boldsymbol{a}$, Mapping of activations of the inhibition of mimicry and the eye contact effect in current study. Areas with red color represent the simple effect of the inhibition of mimicry. Areas with cyan color represent the simple effect of eye contact. White region is the overlap of the two activations. Bold black line represents the functional border between posterior and anterior $\mathrm{mPFC}$ in the review article by Amodio and Frith (2006). $\boldsymbol{b}$, Comparisons of mPFC activation for the mimicry- gaze interaction between the current study and two related studies. The blue region represents the $\mathrm{mPFC}$ activation for interaction in the current study. The red region represents the mPFC activation for the eye contact effect in Kampe et al. (2003). The cyan region represents the mPFC activation for the inhibition of mimicry in Brass et al. (2001). The white region was the overlap of the two past studies. c, Parameter estimates (SPM $\beta \mathrm{s}$ ) for mPFC activations emerged from the interaction contrast in each experimental condition.

also on the set of models tested, we also directly compared the top two models (models 4 and 10) in the RFX analysis. Again, Bayesian model selection revealed a high exceedance probability, favoring model 4 relative to model 10 , of 0.96 .

The left column in Figure 5 illustrates model 4 with the parameter estimates from the FFX and RFX analyses. In this topdown modulation model, the interaction conditions engage mPFC and enhance the connection from mPFC to STS, while hand movement task engages STS and significantly enhances the connection from STS to IFG. As all 12 models were equated for the parameter number of extrinsic inputs, intrinsic connectivity structure, and modulatory effects, the difference in model evidence is only due to model fit, and not to model complexity. The magnitude of these effects can be seen in the rate constants given on each arrow (numbers in brackets are SD). The rate constant refers to the rate of change of neuronal activity (hertz) in one area as induced by another area or by an extrin- sic input. For intrinsic connections, only the rate constants of two connections, $\mathrm{mPFC} \rightarrow$ STS and $\mathrm{mPFC} \rightarrow$ IFG, were significantly different from zero $t_{(17)}=8.20, p<0.001$ and $t_{(17)}=6.29$, $p<0.001$, respectively). Figure 5 also shows the average rate constants in $\mathrm{mPFC}$ induced by interaction (red line) and the average rate constants in STS induced by hand movement task (blue line). Consistent with conventional GLM analysis, the rate of the neuronal activity in $\mathrm{MPFC}$ was significantly enhanced by $0.05 \mathrm{~Hz}$ in interaction conditions, and the neuronal activity in STS was significantly increased by $0.05 \mathrm{~Hz}$ when subjects performed the hand movement task. Moreover, Figure 5 shows the average rate constants for the modulatory effects on the intrinsic connections by the interaction and the task. When eye contact interacts with mimicry, the average rate constant of the connection strength from $\mathrm{mPFC}$ to STS significantly increased from 0.30 to 0.55 in FFX analysis and from 0.29 to 0.46 in RFX analysis (note that the rate constants for intrinsic connection and modulatory effects are additive, thus $0.30+0.25=0.55$ in FFX analysis or $0.29+0.17=0.46$ in RFX analysis). When subjects were engaged in the hand movement task, the connection from STS to IFG had a significant increase in rate constants from near zero to $0.34-0.35$. These parameters for the optimal model highlight three important features of our data. First, the two significant intrinsic connections from $\mathrm{mPFC}$ to STS and from mPFC to IFG suggest that mPFC persistently modulates activity of IFG and STS, regardless of task or stimuli. Second, the hand movement task involves more information propagation between different parts of MNS, especially the connection from the sensory input part (STS) to the motoric part (IFG). Third, the interaction conditions directly influenced $\mathrm{mPFC}$ and enhanced the connection strength from $\mathrm{mPFC}$ to STS. This supports our hypothesis that $\mathrm{mPFC}$ has a central role in the control of mimicry by eye contact and demonstrates that this control is implemented at the input stage of the MNS (STS) rather than at the motor output (IFG).

\section{Discussion}

Our study aimed to reveal the brain systems underlying the control of mimicry by eye contact. The results were compatible with previous findings on the role of the MNS in imitation tasks and the role of mPFC in the inhibition of mimicry and eye contact effect. More importantly, we revealed an interaction between mimicry and eye contact in $\mathrm{mPFC}$, STS, and IFG, suggesting that these three regions are critical in the control of mimicry by eye contact. The subsequent DCM analysis supports a model in which $\mathrm{mPFC}$ is the origin of this control, and the connection strength between mPFC and STS was increased during the inter- 
action. We consider the implications of these results for social cognition.

\section{Mimicry, the eye contact effect, and the inhibition of mimicry}

In accordance with a meta-analysis on action and imitation tasks (Van Overwalle and Baetens, 2009), we report strong activations in IFG, parietal, and temporal regions including IPL and STS when participants performed the hand movement task. In addition, we found that the connection strength from STS to IFG was significantly enhanced by the hand movement task, which supports the crucial role of these two regions in the basic sensorymotor mapping process.

Our results are consistent with previous studies of gaze processing. When participants were engaged in congruent hand movements, a clear eye contact effect emerged in mPFC, STS, and IPL. Activation of $\mathrm{mPFC}$ and STS by direct gaze is consistent with previous fMRI studies in humans (Kampe et al., 2003; Nummenmaa and Calder, 2009; Senju and Johnson, 2009) and single-cell recordings in monkeys (Perrett et al., 1992; Emery, 2000), which together suggest that STS and $\mathrm{mPFC}$ are important for gaze processing. However, just as the behavioral results showed that reaction time in incongruent trials did not change by gaze conditions (Wang et al., 2011), the current fMRI data demonstrated that no brain regions showed more activation to direct gaze than averted gaze in incongruent trials.

Our results are also in line with previous studies of the inhibition of mimicry. In averted gaze conditions, trials requiring inhibition of mimicry engaged portions of the frontal lobe (mPFC, IFG, MFG) and TPJ. This pattern of activation resembles that previously reported by other researchers (Brass et al., 2001, 2005, 2009) in which they used a different stimulus-response compatibility paradigm to study the inhibition of mimicry. Frontal lobe activity during inhibitory processes is also supported by early clinical findings that inhibition of inappropriate responses (e.g., incongruent trials) is a function performed in the frontal lobe (Luria, 1980; Vendrell et al., 1995). However, it is important to note that these frontal brain regions only showed greater activations to incongruent trials in averted gaze conditions, but not in direct gaze conditions. In particular, Figure $4 c$ shows that mPFC is activated in all three direct gaze conditions (direct-congruent, direct-incongruent, and direct-catch), with no reliable differences between them. In our paradigm, gaze information was available near the start of the trial, while the type of mimicry was not apparent until near the end of the trial. Consequently, we suggest that in direct gaze conditions, a strong BOLD signal in mPFC elicited by eye contact dominates the later BOLD signal elicited by the inhibition of mimicry in incongruent trials.

Moreover, it is interesting to see that mPFC regions engaged by the inhibition of mimicry and by eye contact overlap (Fig. $4 a$ ). These results support the claim of Brass et al. (2009) that the inhibition of mimicry overlaps with higher-level social cognitive abilities both at the functional and the neural level. By asking participants to complete a mimicry-inhibition task, a mentalizing task, and a paradigm assessing self-referential judgments, Spengler et al. (2010) found an overlap of activated brain regions in mPFC between the mimicry-inhibition task and the other two social cognition tasks. They also used neuropsychological evidence from patients with prefrontal and temporoparietal lesions to show that the inhibition of mimicry is functionally linked to aspects of social cognitive processing. Here, we found another social cognitive ability — gaze processing — anatomically overlapping with the inhibition of mimicry. Future studies can explore whether gaze processing and the inhibition of mimicry are functionally linked in patients with prefrontal lesions.

\section{The role of mPFC in the control of mimicry by eye contact}

The critical analysis in the present study was of the interaction between mimicry and eye contact. Our previous study demonstrated a behavioral interaction, with direct gaze enhancing mimicry (Wang et al., 2011). Here, our fMRI analysis showed the engagement of mPFC, STS, and IFG in this interaction, which is consistent with our hypothesis that MPFC is a key mediator in how eye contact modulates mimicry. To distinguish the roles of mPFC, STS, and IFG in this interaction, we used a DCM approach that tests the functional connectivity of these regions. The DCM analysis revealed a best-fitting model in which the interaction of mimicry and eye contact activates mPFC and alters the connection strength from $\mathrm{mPFC}$ to $\mathrm{STS}$. This suggests that mPFC is the originator of the eye contact effect, and this region modulates sensory processes in STS, which in turn impacts on IFG. In cognitive terms, mPFC seems to impose top-down control on how actions are processed in STS and IFG. 
Inspection of the parameter estimates in $\mathrm{MPFC}$ provides hints about the underlying mechanisms (Fig. 4). Direct-congruent and averted-incongruent trials are two orthogonal conditions that are believed to have distinct cognitive processes. Strong activation of mPFC in these two conditions (Fig. 4c) suggests that they both demand a strong level of top-down control, with inhibition in the case of the averted-incongruent trials and enhancement in the case of direct-congruent trials. This interpretation assumes that the BOLD signal does not distinguish inhibition from enhancement but just reflects the overall amount of control. This is also evident in the other conditions, where averted-congruent and averted-catch have the lowest MPFC signal, because these two conditions require neither enhancement nor inhibition. The DCM analysis revealed that in the interaction contrast (DirectCong $>$ Direct-Incong) $>$ (Averted-Cong $>$ Averted-Incong) connectivity from $\mathrm{mPFC}$ to STS is increased, which suggests that the same pattern of enhancement and inhibition acts on STS. Unfortunately, the DCM design does not permit a simple effect analysis to confirm this (Stephan et al., 2010). The suggestion that mPFC can both enhance and inhibit mimicry takes our results beyond previous research (Brass et al., 2001), which emphasized only an inhibitory role for $\mathrm{mPFC}$.

The precise anatomical location of the mPFC cluster supports this possibility that this cluster contains distinct neuronal populations with excitatory and inhibitory roles (Fig. 4a). A metaanalysis of task-related neural activations in $\mathrm{MPFC}$ revealed three functional divisions within this region (Amodio and Frith, 2006). The posterior region of $\mathrm{MPFC}$ is activated in response inhibition tasks; the anterior region is responsible for facilitating social cognition tasks involving mentalizing; the orbital region has been linked to the punishment or reward monitoring. When the location of the mPFC cluster in our data was mapped onto these three functional divisions, we found that it was just at the boundary between posterior and anterior mPFC (Fig. 4a). It was more dorsal than other studies simply testing the inhibition of mimicry (Brass et al., 2001) or the eye contact effect (Kampe et al., 2003) (Fig. 4b). As the posterior region involves response inhibition and the anterior region is responsible for facilitating the performance in social cognition tasks, the boundary location supports the possibility that this region of mPFC can both enhance and inhibit mimicry in different contexts. Further studies will be needed to clarify how control signals originating from different neuronal populations within $\mathrm{MPFC}$ related to both BOLD signal and reaction time measures.

\section{Broader implications}

In a broader cognitive framework, our findings support current theories suggesting that mimicry in social contexts requires brain systems beyond the MNS (Southgate and Hamilton, 2008; Brass et al., 2009). In particular, it has been suggested that basic imitation mechanisms implemented in the MNS must be subtly controlled by other social cues (Southgate and Hamilton, 2008). These social cues could act either on the input to the MNS or on the outputs from the MNS (Heyes, 2011). Previous studies of the inhibition of mimicry (Brass et al., 2005, 2009) showed that mPFC mediates modulation to the output of the MNS. Our DCM analysis complements these findings and shows that under different social gazes, $\mathrm{mPFC}$ enhances mimicry by modulating an input to the MNS. More specifically, MNS input is modulated via changing the connection strength from mPFC to STS. Thus, these data are compatible with the model of Heyes (2011), which suggests mimicry can be modulated by changing either input to or output from the MNS but not changing the sensorimotor mapping itself.

Moreover, numerous behavioral studies suggest that imitation is flexible and can be influenced by higher-level cognitive and affective processes, such as mindsets (van Baaren et al., 2009), social status (Cheng and Chartrand, 2003), social attitude (Leighton et al., 2010), strategy (Rumiati et al., 2009), intentionality (Massen and Prinz, 2009), rationality (Gergely et al., 2002), and eye contact (Wang et al., 2011). Several of these processes have previously been linked to $\mathrm{mPFC}$, including responses to social status (Zink et al., 2008), rationality (Brass et al., 2007), and eye contact (Kampe et al., 2003). Our study is the first to investigate the relationship between social sensitivity in $\mathrm{MPFC}$ and the control of imitation, and specifically demonstrated that mPFC controls mimicry on-line by modulating the sensory input of the MNS. In the future, it will be interesting to test whether other higher-level social cognitive processes modulate mimicry through the mediation of mPFC and how mPFC influences the input or output of the MNS.

The present study focused on eye contact because this is a rapidly processed social cue (Senju and Johnson, 2009). Detection of eye contact may be one way to initialize a communication and begin focusing on another person's mental state (Kampe et al., 2003). Our data provide initial evidence that mPFC both responds to eye contact and uses this to control mimicry behavior. This places $\mathrm{mPFC}$ at the core of on-line social interaction, with a critical role in the subtle decision of who and when to imitate. The results further hint that dysfunction of these social evaluation processes in $\mathrm{mPFC}$ would have a detrimental impact on imitation behavior, as seen in autistic spectrum disorder (Southgate and Hamilton, 2008).

To conclude, the results of this study reveal different brain systems underlying the inhibition of mimicry and the eye contact effect. More importantly, we revealed the critical role of mPFC in the interaction between mimicry and gaze. This region acts as a key controller of mimicry by eye contact and performs this function by modulating sensory inputs to action systems. These findings confirm the controlling role of $\mathrm{mPFC}$ in social interaction and demonstrate how different components of the social brain, both the MPFC and the MNS, work together to rapidly control spontaneous mimicry according to the social context.

\section{References}

Adolphs R (2009) The social brain: neural basis of social knowledge. Annual Rev Psychol 60:693-716.

Amodio DM, Frith CD (2006) Meeting of minds: the medial frontal cortex and social cognition. Nat Rev Neurosci 7:268-277.

Bachevalier J, Meunier M, Lu MX, Ungerleider LG (1997) Thalamic and temporal cortex input to medial prefrontal cortex in rhesus monkeys. Exp Brain Res 115:430-444.

Brass M, Heyes C (2005) Imitation: is cognitive neuroscience solving the correspondence problem. Trends Cogn Sci 9:489-495.

Brass M, Zysset S, von Cramon DY (2001) The inhibition of imitative response tendencies. Neuroimage 14:1416-1423.

Brass M, Derrfuss J, von Cramon DY (2005) The inhibition of imitative and overlearned responses: a functional double dissociation. Neuropsychologia 43:89-98.

Brass M, Schmitt RM, Spengler S, Gergely G (2007) Investigating action understanding: inferential processes versus action simulation. Curr Biol 17:2117-2121.

Brass M, Ruby P, Spengler S (2009) Inhibition of imitative behaviour and social cognition. Philos Trans R Soc Lond B Biol Sci 364:2359-2367.

Buccino G, Vogt S, Ritzl A, Fink GR, Zilles K, Freund HJ, Rizzolatti G (2004) Neural circuits underlying imitation learning of hand actions: an eventrelated fMRI study. Neuron 42:323-334.

Carmichael ST, Price JL (1995) Sensory and premotor connections of the 
orbital and medial prefrontal cortex of macaque monkeys. J Comp Neurol 363:642-664.

Catani M, Jones DK, ffytche DH (2005) Perisylvian language networks of the human brain. Ann Neurol 57:8-16.

Chartrand TL, van Baaren R (2009) Human mimicry. Adv Exp Soc Psychol 41:219-274.

Cheng CM, Chartrand TL (2003) Self-monitoring without awareness: using mimicry as a nonconscious affiliation strategy. J Pers Soc Psychol 85:1170-1179.

Emery NJ (2000) The eyes have it: the neuroethology, function and evolution of social gaze. Neurosci Biobehav Rev 24:581-604.

Friston KJ, Harrison L, Penny W (2003) Dynamic causal modelling. Neuroimage 19:1273-1302.

Gergely G, Bekkering H, Kiraly I (2002) Developmental psychology: rational imitation in preverbal infants. Nature 415:755.

Gong G, He Y, Concha L, Lebel C, Gross DW, Evans AC, Beaulieu C (2009) Mapping anatomical connectivity patterns of human cerebral cortex using in vivo diffusion tensor imaging tractography. Cereb Cortex 19:524-536.

Grinband J, Wager TD, Lindquist M, Ferrera VP, Hirsch J (2008) Detection of time-varying signals in event-related fMRI designs. Neuroimage 43:509-520.

Heyes C (2011) Automatic Imitation. Psychol Bull 137:463-483.

Heyes C, Bird G, Johnson H, Haggard P (2005) Experience modulates automatic imitation. Brain Res Cogn Brain Res 22:233-240.

Iacoboni M (2009) Imitation, empathy and mirror neurons. Annu Rev Psychol 60:653-670.

Iacoboni M, Woods RP, Brass M, Bekkering H, Mazziotta JC, Rizzolatti G (1999) Cortical mechanisms of human imitation. Science 286:25262528.

Kampe KK, Frith CD, Frith U (2003) "Hey John": signals conveying communicative intention toward the self activate brain regions associated with "mentalizing," regardless of modality. J Neurosci 23:5258-5263.

Kass RE, Raftery AE (1995) Bayes factors. J Am Stat Assoc 90:773-795.

Kilner JM, Marchant JL, Frith CD (2006) Modulation of the mirror system by social relevance. Soc Cogn Affect Neurosci 1:143-148.

Kuzmanovic B, Georgescu AL, Eickhoff SB, Shah NJ, Bente G, Fink GR, Vogeley K (2009) Duration matters: dissociating neural correlates of detection and evaluation of social gaze. Neuroimage 46:1154-1163.

Leichnetz GR, Astruc J (1976) The efferent projections of the medial prefrontal cortex in the squirrel monkey (Saimiri Sciureus). Brain Res 109:455-472.

Leighton J, Bird G, Orsini C, Heyes CM (2010) Social attitudes modulate automatic imitation. J Exp Soc Psychol 46:905-910.

Liepelt R, Brass M (2010) Top-down modulation of motor priming by belief about animacy. Exp Psychol 57:221-227.

Liepelt R, Crbamon DY, Brass M (2008) What is matched in direct matching? Intentional attribution modulates motor priming. J Exp Psychol Hum Percept Perform 34:578-591.

Luppino G, Rozzi S, Calzavara R, Matelli M (2003) Prefrontal and agranular cingulated projections to the dorsal premotor areas F2 and F7 in the macaque monkey. Eur J Neurosci 17:559-578.

Luria AR (1980) Higher cortical functions in man, 2nd Ed. New York: Basic Books.

Massen C, Prinz W (2009) Movements, actions, and tool-use actions: an ideomotor approach to imitation. Philos Trans R Soc Lond B Biol Sci 364:2349-2358.

Nummenmaa L, Calder AJ (2009) Neural mechanisms of social attention. Trends Cogn Sci 13:135-143.

Penny WD, Stephan KE, Mechelli A, Friston KJ (2004) Comparing dynamic causal models. Neuroimage 22:1157-1172.

Perrett DI, Hietanen JK, Oram MW, Benson PJ (1992) Organization and functions of cells responsive to faces in the temporal cortex. Philos Trans R Soc Lond B Biol Sci 335:23-30.

Press C, Bird G, Walsh E, Heyes C (2008) Automatic imitation of intransitive actions. Brain Cogn 67:44-50.

Rilling JK, Glasser MF, Preuss TM, Ma X, Zhao T, Hu X, Behrens TE (2008) The evolution of the arcuate fasciculus revealed with comparative DTI. Nat Neurosci 11:426-428.

Rizzolatti G, Craighero L (2004) The mirror-neuron system. Annu Rev Neurosci 27:169-192.

Rumiati RI, Carmo JC, Corradi-Dell'Acqua C (2009) Neuropsychological perspectives on the mechanisms of imitation. Philos Trans R Soc Lond B Biol Sci 364:2337-2347.

Senju A, Johnson MH (2009) The eye contact effect mechanisms and development. Trends Cogn Sci 13:127-134.

Southgate V, Hamilton AF (2008) Unbroken mirrors: challenging a theory of autism. Trends Cogn Sci 12:225-229.

Spengler S, von Cramon DY, Brass M (2010) Resisting motor mimicry: control of imitation involves processes central to social cognition in patients with frontal and temporo-parietal lesion. Soc Neurosci 5:401-416.

Stephan KE, Penny WD, Daunizeau J, Moran RJ, Friston KJ (2009) Bayesian model selection for group studies. Neuroimage 46:1004-1017.

Stephan KE, Penny WD, Moran RJ, den Ouden HE, Daunizeau J, Friston KJ (2010) Ten simple rules for dynamic causal modeling. Neuroimage 49:3099-3109.

van Baaren R, Janssen L, Chartrand TL, Dijksterhuis A (2009) Where is the love? The social aspects of mimicry. Philos Trans R Soc Lond B Biol Sci 364:2381-2389.

Van Overwalle F, Baetens K (2009) Understanding others' actions and goals by mirror and mentalizing systems: a meta-analysis. Neuroimage 48:564-584.

Vendrell P, Junqué C, Pujol J, Jurado MA, Molet J, Grafman J (1995) The role of prefrontal regions in the stroop task. Neuropsychologia 33:341-352.

Wang Y, Newport R, Hamilton AF (2011) Eye contact enhances mimicry of intransitive hand movements. Biol Lett 7:7-10.

Zink CF, Tong Y, Chen Q, Bassett DS, Stein JL, Meyer-Lindenberg A (2008) Know your place: neural processing of social hierarchy in humans. Neuron 58:273-283. 